\title{
Determination and Measurement of Color Dissimilarity
}

\author{
I Gede Made Karma
}

\author{
Doctoral Study Program of Engineering Science, Faculty of Engineering, Udayana University, Denpasar, \\ Indonesia \\ *Email : igmkarma@pnb.ac.id
}

\begin{abstract}
There are millions of different colors that exist in this nature. There are colors that can easily be distinguished from other colors, but many are also difficult to distinguish. The ability to distinguish colors is important in the recognition of an object, especially objects in an image. Color can be represented in a three-dimensional RGB color space and each color will have an RGB value with a range of values from 0-255. Conceptually, colors with different RGB values are different colors, but the human eye may not necessarily be able to distinguish them. This study aims to determine and measure the color dissimilarity that can be distinguished by humans. With an experiment comparing a color with a color made with a variation of RGB values, this study resulted that two colors that have a Delta $\mathrm{E}(\Delta \mathrm{E})$ value of 8 can be distinguished by the eye. This result is obtained if both colors have a value of $\Delta \mathrm{R}, \Delta \mathrm{G}$ or $\Delta \mathrm{B}$ of at least 8 , or a value of $\Delta \mathrm{RG}, \Delta \mathrm{RB}$ or $\Delta \mathrm{GB}$ of at least 6 , or a value of $\Delta \mathrm{RGB}$ of at least 5 .
\end{abstract}

Index Terms - color, Delta E, dissimilarity, RGB.

\section{INTRODUCTION}

$\mathrm{E}$ ach object has a variety of characteristics that can be used to recognize the object. The characteristics that are widely used include the shape, texture and color possessed by an object [1]. Based on the shape it has, an object can be recognized and distinguished from other objects [2]. In identifying an object based on its shape, various techniques have been used to represent a form, which is grouped into boundary-based which emphasizes the outer boundaries of an object by ignoring its internal parts, and regions-based which focus more on internal details, other than limit details [3]-[5]. In image recognition based on its texture, images are classified into textured and non-textured images [6]. Compared to the shape and texture characteristics, the color shows better stability and is less sensitive when it comes to rotating and zooming the image [7], [8]. Information about color greatly contributes to the recognition of objects, both to objects that are related to certain colors or not [9]. Color also has a very important role in the process of object recognition by patients with Alzheimer's disease, in addition to other visual forms [10].

Color is one of the most important features in image recognition by humans [11]. With color, we can distinguish objects, places and times. Color is also believed to improve the performance of memory. Color can provide the stimulus coding, remembering and re-recognition an object [12].
Color is identified in three-dimensional color space, which is then modeled into 2 types namely hardware-oriented and user-oriented color space. For types of hardware-oriented color space include RGB (Red Green Blue), CMY (Cyan Magenta Yellow) and YIQ, while user-oriented color space types include HLS (Hue Saturation Luminance), HCV, HSV (Hue Saturation value), HSB, MTM, CIE-LAB, and CIELUV [13]. Most image file formats (JPEG, BMP, GIF) use the RGB color space. RGB color space is defined based on the values of the axes R, G and B [14].

For many years people assumed that the visible spectrum of colors was red, orange, yellow, green, blue, indigo, and violet (ROYGBIV). For people learning and knowing the color theory states that there are main colors, namely red, yellow and blue, which can be mixed to produce other colors. As for those who understand photography assume that the main colors are red, green and blue. The real problem is related to the identification of a color that refers to the name given, while there are many colors that are similar, approaching and appearing to be almost the same [15].

There are millions of colors in this nature. If calculated based on variations in the value of $R, G$ and $B$ that are owned by a color, then there will be $256 \times 256 \times 256$ pieces of color. Each value of $R, G$ and $B$ which is owned by a color varies between $0-255$ [16]. But in reality, even though the color value is different, it turns out that our sense of sight is not 
able to distinguish. In addition, in general, colors that obviously look different, are sometimes identified by the same name. It is also undeniable that there are a number of colors that have similarities with each other, although identified by different names [17]. The color recognition process is carried out in two stages, namely the process of color segmentation and the process of identifying each part of this color. This recognition process uses primary colors, and then these primary colors are segmented and identified by the identity of a particular name [18].

The ability to distinguish one color from another color will also have an impact on the ability to identify an object in an image. The similarity of an object's color, especially between background and foreground can cause fatal errors in identifying an object [19]. Perception, recognition and memory of a color also affect the ability to recognize color or objects [20]. This study aims to determine and measure the amount that can be used to determine that the color is different from other colors, based on visual observations of the eye.

\section{RELATED WORKS}

\section{A. Color Image Quantization}

The actual image color can be expressed as a collection of pixels, each pixel can be expressed as $\{(\mathrm{r}, \mathrm{g}, \mathrm{b}) \mid 0 \leq \mathrm{r}, \mathrm{g}$, $\mathrm{b} \leq 255\}$ in the RGB color space. Image color quantification is done through the process of mapping the color of the image, so that the color of the image can be expressed as $\left\{r_{1}\right.$, $\left.r_{2}, r_{3}, \ldots r_{n}\right\}$ which is the color reference used in image quantification. Furthermore, the process of image color quantification can be done in two steps, namely the design of a color palette and the mapping of image pixels to one of the colors in the palette. Process optimization is intended to minimize the difference in perception between the original image color and the quantified version [21]. In this color quantification process, designing a color palette usually involves only a small part of the millions of colors available. This is done to improve the quality of the perception of the image color [22].

\section{B. Distance and Similarity Measures}

The level of similarity is formulated in an effort to measure the similarity of two objects simply, intuitively and qualitatively. The problem is, the term "similarity" is used in various fields. In other words, the term "similar" can mean different things in different situations / things. Therefore, the formulation of this similarity can vary depending on the object or measurement objective. For the representation of two objects, the level of similarity of objects can be measured based on the distance from the two objects. In measuring the level of similarity of two objects by using distance, the value of a larger distance size indicates that the two objects have a reduced level of similarity [23].

Measurement of the similarity of two colors can be done by performing color transformation from the RGB color space into the color space which is perception is uniform.
The color of the image is then quantified and represented by a subset of fewer colors [24].

\section{Difference in Color of Objects}

Color is believed by many people to have a certain meaning, and is considered always the same or permanent, not influenced by the context in which / when the color is used. In fact, in certain cases such as the color of the product, food, health and others shows that the context influences the meaning of the color [25]. The existence of a color in an image and the context of each color turns out to affect the perception of a color [26].

Our perception of a color is determined by many factors, including the following [27]:

1) The physical characteristics of an object are related to its ability to absorb light,

2) Spectral composition of light sources and their environment,

3) The visual ability of the observer, and experience in observing similar objects,

4) Proximity of objects with other objects.

The difference in perception perceived by the observer is a psychophysical difference determined by the actual observation of the two samples. This difference occurs because of different stimuli from the colors represented by points in space. To measure the ability of the eye to distinguish between colors, a metric has been developed known as Delta $\mathrm{E}(\Delta \mathrm{E})$ which shows the Euclidean distance from two coordinates. The color difference $\Delta \mathrm{E}$ is calculated as the distance between the observed color points [28]. This $\Delta \mathrm{E}$ calculation is influenced by the color model used. Delta $\mathrm{E}(\Delta \mathrm{E})$ has been used as a standard calculation related to human visual assessment of the differences in the two colors [29]. In the industrial world, this $\Delta \mathrm{E}$ has been used to measure the deviation of a color from a product and determine the allowable deviation tolerance [30], [31].

The $\Delta \mathrm{E}$ concept was introduced by the International Commission on Illumination (CIE) as a standard in the fields of colorimetry, photometry, and imaging. The reference set as a guide in determining the perception of color, based on the amount of $\Delta \mathrm{E}$ values in the range $0-100$ is presented in Table I [32].

TABLE I

PERCEPTION BASED ON $\triangle \mathrm{E}$

\begin{tabular}{|l|l|}
\hline \multicolumn{1}{|c|}{$\Delta \mathbf{E}$} & Perception \\
\hline$<=1.0$ & Not perceptible by human eyes. \\
\hline $1-2$ & Perceptible through close observation. \\
\hline $2-10$ & Perceptible at a glance. \\
\hline $11-49$ & Colors are more similar than opposite \\
\hline 100 & Colors are exact opposite \\
\hline
\end{tabular}

\section{RESEARCH METHODOLOGY}

To determine the color dissimilarity that can be identified by the senses of vision, there will be a number of experiments. Experiments carried out by comparing a specific color with other colors that are variations of these 
colors. Other colors that are variations of this color is a color that is made by changing the value of the $R, G$ or $B$ of the color.

The research procedure is:

1) Choose a color with certain $R, G$ and $B$ values.

2) Compare this color with 30 (thirty) variations of this color with different values of $\mathrm{R}, \mathrm{G}$ and $\mathrm{B}$ in the range 0 29.

3) Repeat step 2, by comparing this color with 30 (thirty) variations in the value differences $(R$ and $G),(R$ and $B)$, and ( $\mathrm{G}$ and $\mathrm{B})$ with a range of $0-29$.

4) Repeat step 2, by comparing this color with 30 (thirty) variation of the difference in value ( $R$ and $G$ and $B$ ) with a range of 0-29.

5) Make observations on the dissimilarity in color display for each comparison of these values.

This research was conducted using a MATLAB R2017b program, with a flowchart as presented in Fig. 1.

\section{EXPERIMENT RESULT}

From the observations made on the results of program execution, the results obtained are presented in Table II. These results are obtained by selecting colors with the same R, $G$ and $B$ values i.e. 30 . Except for the image 1 in Table II, the image of the results of this experiment seems to be sorted into two parts, half above and half below. The top half contains colors with fixed values of $R, G$ and $B$, while the bottom contains colors with a variation of $R, G$ and $B$ values of 30 pieces, ranging from $0-29$.

In sequence, the results images in Table II are:

1) The bottom part contains colors that have the same $R, G$ and $B$ values as the $R, G$ and $B$ values of the color above it.

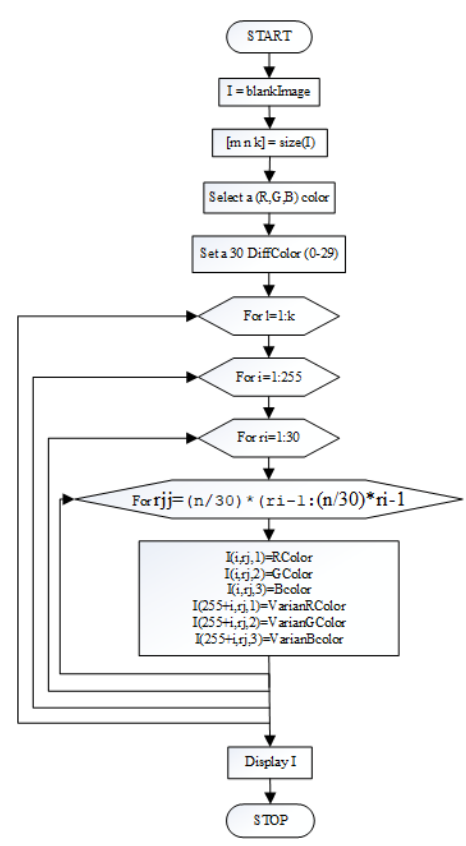

Fig. 1. Flowchart of the program used in observing color dissimilarity.

TABLE II

RESULTS OF OBSERVATION 1
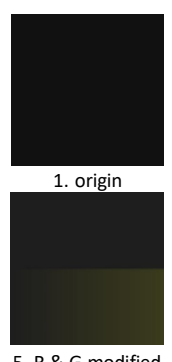

5. R \& G modified
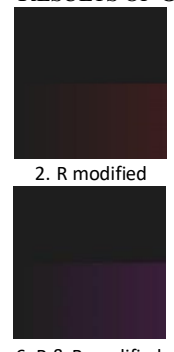

6. R \& B modified

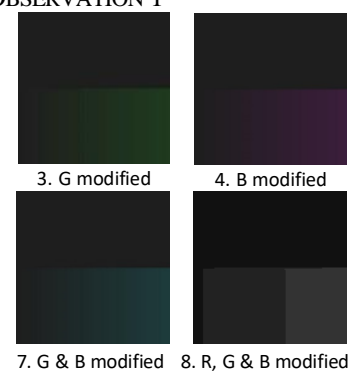

2) The bottom part contains a color that has a different $R$ value than the $R$ value of the color above it, while the $G$ and $B$ values are the same.

3) The bottom part contains a color that has a different $G$ value than the $G$ value of the color above it, while the $R$ and $\mathrm{B}$ values are the same.

4) The bottom part contains a color that has a different $B$ value than the $B$ value of the color above it, while the $R$ and $\mathrm{G}$ values are the same.

5) The bottom part contains a color that has a different $R$ and $G$ value than the $R$ and $G$ value of the color above it, while the $B$ values are the same.

6) The bottom part contains a color that has a different $R$ and $B$ value than the $R$ and $B$ value of the color above it, while the $G$ values are the same.

7) The bottom part contains a color that has a different $G$ and $B$ value than the $G$ and $B$ value of the color above it, while the $\mathrm{R}$ values are the same.

8) The bottom part contains a color that has a different $R$, $G$ and $B$ value than the $R, G$ and $B$ value of the color above it.

Because it has a different combination of $\mathrm{R}, \mathrm{G}$ or B values, it is understandable that the 2-8 experimental images on the bottom have a different color display with the colors on the top, and colors from left to right.

The same pattern was also obtained from the results of the experiment by using a choice of colors with different $R, G$ and $\mathrm{B}$ values. The difference is only in the color display.

The focus of our observations is the color display in the upper half and the lower half, from left to right. It appears that there are differences in color display, ranging from vague to very clear. The greater the difference in the value of the bottom color RGB with the color of the top, the clearer the difference. The clarity of the existing color differences have the same pattern in the results of 2-8 experiments. That is, the color difference between the two can already occur due to differences in one of the RGB values or a combination of two or three values.

In plain view, the color difference that occurred in the results of this experiment (2-8) was not as fully visible to the eye. Apart from several factors that have been explained before, the eye does have limitations in distinguishing one color from another [33]. The question is, how much difference should the RGB value of a color pair have, so that the color can be easily distinguished.

As seen in the results of experiments 2-8, the difference in 
color in the left third of each image is almost indistinguishable from the eye. Based on experimental data, the difference in the RGB value of the left third of the image is $1-10$. Means, colors with differences in RGB values of less than 10 , are already visible but not very clear can be distinguished by the eye. To measure and determine the magnitude of the difference in RGB values that must be owned by color, so that it can easily be distinguished by the eye, further experiments will be carried out. The experiment will be carried out with the same procedure that is 15 times, with differences in values ranging from 1 to 15 on the $R$ value only. The selection of the tested values may actually be free, because previous experiments have shown that differences can occur due to one or a combination of all RGB values.

Table III that displays the results of experiments with differences in the value of $\mathrm{R}$ of 1-15 shows that the results of the difference in the value of $\mathrm{R}$ of $1-5$, there is practically no difference. Even if there are differences, the eye is practically very difficult to distinguish. At a difference of $R$ values of 6 and 7 , the difference begins to appear, but it is not clear. The difference starts clearly visible in the difference in the value of $\mathrm{R}$ of 8 and above. The ability of the eye to distinguish these colors can of course differ from one another, because other factors can also influence it.

Based on observations from the results of this experiment, we can then do calculations to find out the magnitude of the difference in the value of a color so that it can be clearly distinguished by the eye. This difference value can then be used as an indicator of color differentiation.

Calculation of the difference between the two colors expressed by the values of $\mathrm{R}, \mathrm{G}$, and $\mathrm{B}$, although less accurate due to the uniformity of the color space, can still be determined by the Euclidean distance formula [34]:

$\Delta E_{R G B}=\sqrt{ } \Delta R^{2}+\Delta G^{2}+\Delta B^{2}$

Because the eyes have different sensitivity to changes in RGB values and lighting levels and the lightness / darkness of a color, the formula (1) is refined to:

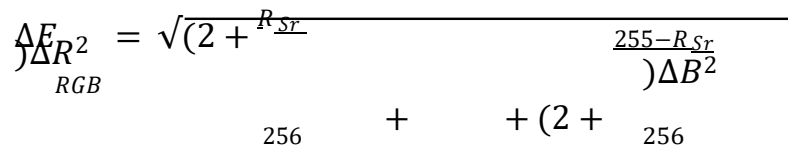

TABLE IV

$\Delta \mathrm{E}_{\mathrm{RgB}}$ CAlCulations Results

\begin{tabular}{|c|c|c|c|c|c|c|c|c|c|c|c|c|c|c|}
\hline \multirow[b]{2}{*}{ ? } & \multicolumn{7}{|c|}{ Fi mula (1) } & \multicolumn{7}{|c|}{$F_{i}$ mula (2) } \\
\hline & $\mathrm{R}$ & $\mathrm{G}$ & B & RG & RB & GB & RGB & $\mathrm{R}$ & G & B & RG & RB & GB & RGB \\
\hline 1 & 1 & 1 & 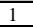 & 1.41 & 1.41 & 1.41 & 1.73 & 1.46 & 2.00 & \begin{tabular}{|l|}
1.70 \\
\end{tabular} & \begin{tabular}{|l|}
2.47 \\
\end{tabular} & 2.24 & 2.62 & 3.33 \\
\hline 2 & 2 & 2 & 2 & 2.83 & 2.83 & 2.83 & 3.46 & 2.91 & \begin{tabular}{|l|}
4.00 \\
\end{tabular} & \begin{tabular}{|l|}
2.35 \\
\end{tabular} & \begin{tabular}{|l|}
4.95 \\
\end{tabular} & 3.74 & \begin{tabular}{|l|l|}
4.64 \\
\end{tabular} & 6.20 \\
\hline 3 & 3 & 3 & 3 & 4.24 & 4.24 & \begin{tabular}{|l|}
4.24 \\
\end{tabular} & 5.20 & \begin{tabular}{|l|}
4.37 \\
\end{tabular} & \begin{tabular}{|l|l|}
6.00 \\
\end{tabular} & \begin{tabular}{|l|}
3.14 \\
\end{tabular} & 7.42 & 5.38 & \begin{tabular}{|l|}
6.77 \\
\end{tabular} & \begin{tabular}{|l|}
9.17 \\
\end{tabular} \\
\hline 4 & 4 & 4 & 4 & 6 & 5.66 & 5.66 & \begin{tabular}{|l|}
6.93 \\
\end{tabular} & 5.83 & \begin{tabular}{|l|}
8.00 \\
\end{tabular} & \begin{tabular}{|l|}
3.99 \\
\end{tabular} & 9.90 & 7.07 & 8.94 & 12.17 \\
\hline 5 & 5 & 5 & 5 & 7.07 & 7.07 & 7.07 & \begin{tabular}{|l|}
8.66 \\
\end{tabular} & 7.29 & 10.00 & \begin{tabular}{|l|}
4.87 \\
\end{tabular} & 12.38 & \begin{tabular}{|l|}
8.77 \\
\end{tabular} & \begin{tabular}{|l|}
11.12 \\
\end{tabular} & 15.17 \\
\hline 6 & 6 & 6 & 6 & 49 & 8.49 & \begin{tabular}{|l|}
8.49 \\
\end{tabular} & 10.39 & 8.75 & 12.00 & \begin{tabular}{|l|}
5.76 \\
\end{tabular} & 14.85 & 10.48 & 13.31 & 18.19 \\
\hline 7 & 7 & 7 & 7 & 9.90 & 9.90 & \begin{tabular}{|l|}
9.90 \\
\end{tabular} & 12.12 & 10.22 & 14.00 & \begin{tabular}{|l|l|}
6.66 \\
\end{tabular} & 17.33 & 12.20 & 15.50 & 21.21 \\
\hline 8 & 8 & 8 & 8 & 1 & 11.31 & 11.31 & 13.86 & 11.68 & 16.00 & \begin{tabular}{|l|}
7.57 \\
\end{tabular} & 19.81 & 13.92 & 17.70 & 24.23 \\
\hline 9 & 9 & 9 & 9 & 2.73 & 12.73 & 12.73 & 15.59 & 13.15 & 18.00 & \begin{tabular}{|l|}
8.47 \\
\end{tabular} & 22.29 & 15.64 & 19.89 & 27.25 \\
\hline 10 & 10 & 10 & 10 & 4.14 & 14.14 & 14.14 & 17.32 & 14.62 & 20.00 & 9.38 & 24.77 & 17.37 & 22.09 & 30.28 \\
\hline 11 & 11 & 11 & 11 & 15.56 & 15.56 & 15.56 & 19.05 & 16.09 & 22.00 & \begin{tabular}{|l|} 
\\
\end{tabular} & 27.25 & 19.09 & 24.28 & 33.31 \\
\hline 12 & 12 & 12 & 12 & 16.97 & 16.97 & 16.97 & 20.78 & 17.56 & 24.00 & \begin{tabular}{|l|}
11.19 \\
\end{tabular} & 29.74 & 20.82 & 26.48 & 36.34 \\
\hline 13 & 13 & 13 & 13 & 18.38 & 18.38 & 18.38 & 22.52 & 19.03 & 26.00 & 12.09 & 32.22 & 22.55 & 28.67 & 39.37 \\
\hline 14 & 14 & 14 & 14 & 19.80 & 19.80 & \begin{tabular}{|l|}
19.80 \\
\end{tabular} & 24.25 & 20.50 & \begin{tabular}{|l|}
28.00 \\
\end{tabular} & \begin{tabular}{|l|}
13.00 \\
\end{tabular} & 34.70 & 24.27 & \begin{tabular}{|l|}
30.87 \\
\end{tabular} & 42.40 \\
\hline 15 & 15 & 15 & 15 & 21.21 & 21.21 & \begin{tabular}{|l|}
21.21 \\
\end{tabular} & \begin{tabular}{|l|}
25.98 \\
\end{tabular} & 21.98 & 30.00 & \begin{tabular}{|l|}
13.90 \\
\end{tabular} & 37.19 & 26.00 & 33.06 & 45.44 \\
\hline
\end{tabular}

The calculation results in Table IV show that the greater the $\Delta$ color, the greater the $\triangle E R G B$, both in calculations using formulas (1) or (2). When referring to the results of observations in the second experiment that colors can be clearly distinguished when the value of $\Delta \mathrm{R}$ is 8 , then the color can already be distinguished when it has a value of $\triangle \mathrm{ERGB}$ of 8 for formula (1) or 11.68 for formula (2). If the magnitude of the $\Delta \mathrm{E}_{\mathrm{RGB}}$ value is then used as the basis for determining the color dissimilarity, then the color dissimilarity can be obtained in various combinations of $\triangle \mathrm{RGB}$. The $\Delta \mathrm{E}_{\mathrm{RGB}}$ value of 8 with formula (1) can be obtained at the value of $\Delta R, \Delta G$ and $\Delta B$ is 8 , the value of $\Delta \mathrm{RG}, \Delta \mathrm{RB}$ and $\Delta \mathrm{GB}$ is 6 , or the value of $\Delta \mathrm{RGB}$ is 5 . Whereas with the formula (2), $\Delta \mathrm{E}_{\mathrm{RGB}}$ value of 11.68 can be obtained at $\Delta \mathrm{R}$ value is $8, \Delta \mathrm{G}$ value is $6, \Delta \mathrm{B}$ value is $13, \Delta \mathrm{RG}$ value is $5, \Delta \mathrm{RB}$ value is $7, \Delta \mathrm{GB}$ value is 6 , or $\Delta \mathrm{RGB}$ value is 4 . This Delta $(\Delta)$ value is the minimum value that color must have.

\section{CONCLUSION AND FUTURE WORK}

Although many factors can affect the ability of the eye to distinguish colors, color dissimilarity can be clearly determined when there are differences in each component of a minimum RGB value of 8 , or a combination of two components of an RGB value of at least 6 , or a combination of three components of a minimum RGB value of 5 This color dissimilarity is indicated when the color has a minimum $\Delta \mathrm{E}_{\mathrm{RGB}}$ value of 8 . The greater the $\Delta \mathrm{E}_{\mathrm{RGB}}$ value, the dissimilarity is clearer, and vice versa. The first experiment showed that the calculation of the value $\Delta E_{R G B}$ with formula (2) was less acceptable as an indicator to assess the color

dissimilarity.

The results of this study can then be used as a basis for determining and developing color segmentation techniques and at the same time developing methods for identifying objects in an image.

\section{ACKNOWLEDGMENT}

The author would like to thank and appreciate as much as possible to all parties, especially family members, for their support, so that this research can be completed well. 


\section{REFERENCES}

[1] C. S. Gode and A. N. Ganar, "Image Retrieval by Using Colour, Texture adn Shape Features," Int. J. Adv. Res. Electr. Electron. Instrum. Eng., vol. 3, no. 4, pp. 8637-8644, 2014.

[2] M. Safar, C. Shahabi, and X. Sun, "Image retrieval by shape: a comparative study," in Multimedia and Expo, 2000. ICME 2000. 2000 IEEE International Conference on, 2000, vol. 1, pp. 141-144.

[3] A. Amanatiadis, V. G. Kaburlasos, A. Gasteratos, and S. E. Papadakis, "A comparative study of invariant descriptors for shape retrieval," in Imaging Systems and Techniques, 2009. IST'09. IEEE International Workshop on, 2009, pp. 391-394.

[4] C. Shahabi and M. Safar, "An experimental study of alternative shapebased image retrieval techniques," Multimed. Tools Appl., vol. 32, no. 1, pp. 29-48, 2007.

[5] D. Zhang and G. Lu, "An integrated approach to shape based image retrieval," Proc. 5th Asian Conf. Comput. Vis. (ACCV), Melbourne, Aust., 2002.

[6] J. Li, J. Z. Wang, and G. Wiederhold, "Classification of Textured and Non-Textured Images Using Region Segmentation," in IEEE International Conference on Image Processing (ICIP), 2000, pp. 754 757.

[7] G. Mandloi, "A Survey on Feature Extraction Techniques for Color Images," Int. J. Comput. Sci. Inf. Technol., vol. 5, no. 3, pp. 46154620, 2014.

[8] S. Kumar and E. A. Chauhan, "A survey on feature extraction techniques for color images."

[9] I. Bramão, A. Reis, K. M. Petersson, and L. Faísca, "The role of color information on object recognition: A review and meta-analysis," Acta Psychol. (Amst)., vol. 138, no. 1, pp. 244-253, 2011.

[10] R. L. Adlington, K. R. Laws, and T. M. Gale, "Visual processing in Alzheimer's disease: Surface detail and colour fail to aid object identification," Neuropsychologia, vol. 47, no. 12, pp. 2574-2583, 2009

[11] K. Yang, S. Gao, C. Li, and Y. Li, "Efficient color boundary detection with color-opponent mechanisms," in Proceedings of the IEEE conference on computer vision and pattern recognition, 2013, pp. 2810-2817.

[12] M. A. Dzulkifli and M. F. Mustafar, "The influence of colour on memory performance: A review," Malaysian J. Med. Sci. MJMS, vol. 20, no. 2, p. 3, 2013.

[13] P. Kaur, S. Thakral, and M. Singh, "Color Based Image Retrieval System," IOSR J. Comput. Eng., 2012.

[14] S. V Sakhare and V. G. Nasre, "Design of feature extraction in content based image retrieval (CBIR) using color and texture," Int. J. Comput. Sci. Informatics, vol. 1, no. 2, pp. 57-61, 2011.

[15] M. Wallin and R. Meganck, "Mapping Color," 2014.

[16] L. Brun and A. Trémeau, "Color quantization," Digit. Color Imaging Handb., pp. 589-638, 2003.

[17] A. BYRNE, "Color and Similarity," Philos. Phenomenol. Res., vol. 66, no. 3, pp. 641-665, May 2003.

[18] D. Senthamaraikannan, S. Shriram, and J. William, "Real time color recognition," Int. J. Innov. Res. Electr. Electron. Instrum. Control Eng., vol. 2, no. 3, 2014.

[19] X. Zhang and J. Yang, "The analysis of the color similarity problem in moving object detection," Signal Processing, 2009.

[20] H. Zimmer and A. Steiner, "Colour specificity in episodic and in perceptual object recognition with enhanced colour impact," Eur. J. Cogn. Psychol., vol. 15, no. 3, pp. 349-370, 2003.

[21] S.-C. Cheng and C.-K. Yang, "A fast and novel technique for color quantization using reduction of color space dimensionality," Pattern Recognit. Lett., vol. 22, no. 8, pp. 845-856, 2001.

[22] M. Hassan and C. Bhagvati, "Structural Similarity Measure for Color Images," Int. J. Comput. Appl., vol. 43, no. 14, pp. 7-12, Apr. 2012.

[23] M. S. Mulekar and C. S. Brown, "Distance and Similarity Measures," in Encyclopedia of Social Network Analysis and Mining, New York, NY: Springer New York, 2017, pp. 1-16.

[24] A. Mojsilovic, H. Hu, and E. Soljanin, "Extraction of perceptually important colors and similarity measurement for image matching, retrieval and analysis," IEEE Trans. Image Process., vol. 11, no. 11, pp. 1238-1248, Nov. 2002.

[25] S. Won and S. Westland, "Colour meaning and context," Color Res. Appl., vol. 42, no. 4, pp. 450-459, 2017.

[26] B. Oicherman, "To develop a procedure for visual evaluation of colour differences between printed complex images," 2003.
[27] M. Wojciech and T. Maciej, "Color difference Delta E - A survey," Mach. Graph. Vis., 2011.

[28] D. H. Brainard, "Color appearance and color difference specification," Sci. Color, vol. 2, pp. 191-216, 2003.

[29] L. Hadimani and N. Mittal, "Development of a computer vision system to estimate the colour indices of Kinnow mandarins," J. Food Sci. Technol., vol. 56, no. 4, pp. 2305-2311, 2019.

[30] ViewSonic, "Technical Introduction," 2010. [Online]. Available: https://www.viewsonic.com/uk/products/lcd/pdf/ti_delta_E.pdf.

[31] K. Minolta, "Identifying Color Differences Using $\mathrm{L}^{*} \mathrm{a}^{*} \mathrm{~b}^{*}$ or $\mathrm{L}^{*} \mathrm{C}^{*} \mathrm{H}^{*}$ Coordinates," $2016 . \quad$ [Online]. Available: https://sensing.konicaminolta.us/blog/identifying-color-differencesusing-l-a-b-or-l-c-h-coordinates/.

[32] Z. Schuessler, "Delta E 101," 2016. [Online]. Available: http://zschuessler.github.io/DeltaE/learn/.

[33] S. Mecklenbräuker, A. Hupbach, and W. Wippich, "What colour is the car? Implicit memory for colour information in children," Q. J. Exp. Psychol. Sect. A, vol. 54, no. 4, pp. 1069-1086, 2001.

[34] W. S. Mokrzycki and M. Tatol, "Colour difference $\Delta$ E-A survey," Mach. Graph. Vis., vol. 20, no. 4, pp. 383-411, 2011. 